\title{
Comportamiento a tracción a temperaturas ambiente y elevadas de nuevos composites basados en aleaciones hipereutécticas de $\mathrm{Al}_{-\mathrm{Si}}{ }^{(\bullet)}$
}

\author{
J. Valer ${ }^{(*)}$, J.M. Rodríguez ${ }^{(*)}$ y J.J. Urcola ${ }^{(*)}(\dagger)$ \\ Resumen En este trabajo se muestra la mejora obtenida en la resistencia a la tracción a temperatura ambiente y \\ a elevadas temperaturas de aleaciones hipereutécticas de Al-Si producidas por una combinación de \\ un proceso de solidificación rápida y del conformado en estado semisólido, en comparación con \\ aleaciones obtenidas por procedimientos convencionales de inyección en estado líquido. Se han \\ estudiado las aleaciones $\mathrm{Al}-25 \% \mathrm{Si}-5 \% \mathrm{Cu}, \mathrm{Al}-25 \% \mathrm{Si}-5 \% \mathrm{Cu}-2 \% \mathrm{Mg}$ y $\mathrm{Al}-30 \% \mathrm{Si}-5 \% \mathrm{Cu}$, relacionando \\ sus parámetros microestructurales con las resistencias a tracción obtenidas, y se han comparado con \\ las aleaciones binarias $\mathrm{Al}-20 \% \mathrm{Si}, \mathrm{Al}-36 \% \mathrm{Si}$ y Al-50\%Si. La aleación Al-25\%Si-5\% Cu se ha \\ ensayado antes y después del conformado en estado semisólido, lo que ha permitido conocer la \\ diferencia en el comportamiento de la aleación como consecuencia de la distinta microestructura. \\ También se comparan las propiedades obtenidas en estas aleaciones con las que presentan \\ composites de aleaciones de aluminio reforzados con partículas de $\mathrm{SiC}$ y procesados por métodos \\ similares.
}

Palabras clave: Aleaciones hipereutécticas Al-Si. Conformado semisólido. Comportamiento mecánico.

\section{Tensile behaviour at room and high temperatures of novel metal matrix composites based on hypereutectic Al-Si alloys}

\begin{abstract}
This work shows the improvement obtained on tensile stress at room and high temperatures of hypereutectic Al-Si alloys. These alloys are produced by a combination of spray-forming, extrusion and thixoforming process, in comparison with conventional casting alloys. $\mathrm{Al}-25 \% \mathrm{Si}-5 \% \mathrm{Cu}, \mathrm{Al}-$ $25 \% \mathrm{Si}-5 \% \mathrm{Cu}-2 \% \mathrm{Mg}$ and $\mathrm{Al}-30 \% \mathrm{Si}-5 \% \mathrm{Cu}$ alloys have been studied relating their microstructural parameters with tensile stress obtained and comparing them with conventional $\mathrm{Al}-20 \% \mathrm{Si}, \mathrm{Al}-36 \% \mathrm{Si}$ and $\mathrm{Al}-50 \% \mathrm{Si}$ alloys. $\mathrm{Al}-25 \% \mathrm{Si}-5 \% \mathrm{Cu}$ alloy was tested before and after semi-solid forming, in order to distinguish the different behaviour of this alloy due to the different microstructure. The properties obtained with these alloys were also related to $\mathrm{Al}-\mathrm{SiC}$ composites formed by similar processes.
\end{abstract}

Keywords: Hypereutectic Al-Si alloys. Thixoforming process. Mechanical behaviour

\section{INTRODUCCIÓN}

Las aleaciones hipereutécticas de $\mathrm{Al}-\mathrm{Si}$ son muy interesantes para las industrias del automóvil y

(•) Trabajo recibido el día 25 de marzo de 1996.

(*) Centro de Estudios e Investigaciones Técnicas de Guipúzcoa y E.S.I.I. de San Sebastián. Po de Manuel Lardizábal, 15. 20009-San Sebastián (España).

(†) El Prof. J.J. Urcola falleció el día 12 de agosto de 1996 como consecuencia de un accidente en Jaca (Huesca, España). aeronáutica, debido a su ligereza, sus aceptables propiedades específicas a temperatura ambiente y a elevadas temperaturas, a su bajo coeficiente de expansión térmica y a su excelente resistencia al desgaste. Sin embargo, cantidades de silicio superiores al $20 \%$ en masa requieren elevadas temperaturas de procesamiento por métodos convencionales $\mathrm{y}$, además, se obtienen unas pobres propiedades mecánicas como consecuencia de la presencia de partículas de silicio de gran tamaño y de porosidad (1). 
El interés en estas aleaciones ha impulsado el desarrollo de nuevas tecnologías de producción que eviten estos problemas, dentro de las cuales se encuentra una combinación de un proceso de solidificación rápida (Proceso Osprey) y del conformado en estado semisólido (Thixoforming) (2).

Mediante esta tecnología se obtienen microestructuras finas y homogéneas que dan lugar a una notable mejora de las propiedades mecánicas en comparación a las obtenidas en aleaciones producidas convencionalmente.

La presencia de partículas de silicio primario distribuidas en una matriz de aluminio convierte a estas aleaciones en composites de matriz metálica. Debido a esta característica, el comportamiento mecánico de estas aleaciones puede estudiarse siguiendo las teorías utilizadas para el estudio de composites reforzados con partículas ( 3 y 4 ).

En los composites reforzados con partículas (aleaciones de aluminio reforzadas con partículas cerámicas), la iniciación de la fractura del material se produce por fractura de las partículas, por la fractura a través de la intercara entre las partículas y la matriz o por fractura de la matriz, dependiendo de la naturaleza de las partículas y de la matriz, de la fracción en volumen de partículas, de su tamaño, de su distribución en la matriz o del tratamiento térmico del material $(3,5$ y 6$)$.

A continuación se van a presentar los resultados de los ensayos de tracción a temperatura ambiente obtenidos en aleaciones hipereutécticas binarias de $\mathrm{Al}-\mathrm{Si}$ producidas convencionalmente por inyección líquida, y su comparación con las obtenidas en las nuevas aleaciones hipereutécticas procesadas en estado semisólido.

Puesto que las aleaciones hipereutécticas se usan en aplicaciones donde se requiera un aceptable comportamiento a elevada temperatura, se presentan los resultados obtenidos en las aleaciones conformadas en estado semisólido, ensayadas a 100, 200 y $300{ }^{\circ} \mathrm{C}$.

\section{TÉCNICAS EXPERIMENTALES}

Las aleaciones hipereutécticas binarias Al$20 \% \mathrm{Si}, \mathrm{Al}-36 \% \mathrm{Si}$ y $\mathrm{Al}-50 \% \mathrm{Si}$ se han producido por inyección convencional en la empresa Fagor Ederlan, en un molde especialmente fabricado para la obtención de probetas de tracción de sección circular $(\varnothing=12 \mathrm{~mm})$ y longitud de la zona recta de 80 $\mathrm{mm}$. Estas probetas se ensayaron en una máquina Tinius-Olsen con registrador gráfico. Se utilizó un extensómetro de $50 \mathrm{~mm}$.

Las nuevas aleaciones hipereutécticas se ensayaron en una máquina Instron, bajo control de posición y con una velocidad de desplazamiento del cabezal de $0,5 \mathrm{~mm} / \mathrm{min}$. Estas aleaciones se ensaya- ron en estado atomizado y extruido (producidas en Osprey) y en la condición posterior al conformado en estado semisólido (realizado en la Universidad de Sheffield). Las probetas utilizadas fueron de sección rectangular $\left(6 \times 3 \mathrm{~mm}^{2}\right)$ con una zona recta de $25 \mathrm{~mm}$ y se ensayaron con una celda de carga de 10 $\mathrm{kN}$. El alargamiento de las probetas se determinó con la lectura de un extensómetro de $10 \mathrm{~mm}$, a partir de las medidas de carga-desplazamiento obtenidas de la curva tensión real frente a deformación real. Todas las probetas ensayadas fueron analizadas fractográficamente en un microscopio electrónico de barrido Philips. Esto permitió desechar todas aquellas probetas que presentasen en la superficie de fractura una elevada porosidad, particularmente en el caso del material conformado en estado semisólido.

Los ensayos de tracción en caliente se efectuaron en una máquina Instron con cámara ambiental de atmósfera de aire, utilizando un extensómetro refrigerado por agua. Las temperaturas de ensayo fueron de $100,200,300{ }^{\circ} \mathrm{C}$, con un tiempo de mantenimiento de media hora a cada temperatura. Solamente la aleación $\mathrm{Al}-25 \% \mathrm{Si}-5 \% \mathrm{Cu}$ se ensayó en la condición atomizada y extruida y en la condición final, lo que ha permitido conocer el comportamiento de la aleación en ambas condiciones. Las aleaciones $\mathrm{Al}-25 \% \mathrm{Si}-5 \% \mathrm{Cu}-2 \% \mathrm{Mg}$ y $\mathrm{Al}-30 \% \mathrm{Si}-$ $5 \% \mathrm{Cu}$ se ensayaron después del conformado en estado semisólido.

Finalmente, se ha medido la cantidad de silicio en las superficies de fractura mediante la preparación de cortes longitudinales de las probetas ensayadas, que permiten observar el perfil de fractura. La medida de la cantidad de silicio en el perfil de fractura se realizó mediante un analizador de imágenes.

\section{RESULTADOS Y DISCUSIÓN}

\subsection{Ensayos de tracción a temperatura ambiente}

\subsubsection{Aleaciones convencionales}

Los resultados de los ensayos de tracción de las aleaciones convencionales se muestran en la tabla I. Como se puede observar, tanto la resistencia a la tracción $\left(\sigma_{\text {UTS }}\right)$ como la deformación a fractura disminuyen con el incremento de la cantidad de silicio. Además, excepto en la aleación con un $20 \%$ Si en masa, en el resto de los casos no se alcanza el límite elástico, produciéndose la fractura en el tramo ascendente de la curva tensión-deformación.

Estos pésimos resultados se pueden atribuir a la presencia de una importante cantidad de porosidad, de gran tamaño, en el eje longitudinal de las 
TABLA I.- Resultados de los ensayos de tracción de las aleaciones convencionales

TABLE I.- Tensile test results of conventional alloys

\begin{tabular}{|c|c|c|c|}
\hline \multicolumn{1}{|c|}{ Aleación } & $\begin{array}{c}\sigma_{\mathrm{Y}}, \\
\mathrm{MPa}\end{array}$ & $\begin{array}{c}\sigma_{\mathrm{UTS}}, \\
\mathrm{MPa}\end{array}$ & $\begin{array}{c}\text { Deformación, } \\
\%\end{array}$ \\
\hline \multirow{3}{*}{$\mathrm{Al}-20 \% \mathrm{Si}$} & 137 & 189 & 1,2 \\
& 128 & 178 & 1 \\
& 117 & 157 & 0,9 \\
\hline $\mathrm{Al}-36 \% \mathrm{Si}$ & - & 118 & - \\
& - & 81 & - \\
\hline $\mathrm{Al}-36 \% \mathrm{Si}-0,5 \% \mathrm{Sr}$ & - & 64,5 & - \\
& - & 43 & - \\
\hline $\mathrm{Al}-50 \% \mathrm{Si}$ & - & 44 & - \\
& - & 32 & - \\
\hline
\end{tabular}

probetas. La porosidad se debe a varios motivos: en primer lugar al gas atrapado como consecuencia de la turbulencia del metal líquido al ser introducido en el molde; otra fuente de porosidad se debe a la aparición de una cantidad elevada de partículas de silicio primario de gran tamaño que impiden una buena alimentación de las zonas interdendríticas. Esto último aumenta con la cantidad de silicio.

La porosidad también puede deberse a la contracción por solidificación (rechupe). Sin embargo, a medida que aumenta la cantidad de silicio en la aleación disminuye la contracción por solidificación, situándose, para contenidos de silicio de 20-24\%, entre $1-2 \%$ (7). La presencia de cantidades elevadas de silicio contribuye favorablemente a la disminución del rechupe, debido a que el silicio es uno de los pocos elementos que aumentan su volumen al solidificar (8).

Por otra parte, las pobres propiedades mecánicas de las aleaciones hipereutécticas producidas por inyección convencional también se pueden atribuir a la presencia de partículas de silicio de gran tamaño y de morfología compleja, que actúan como concentradoras de tensiones y originan la fractura frágil del material con cargas y ductilidad muy bajas.

En la figura 1 se muestran unas fractografías en las que se pueden observar varios de los detalles comentados: partículas de gran tamaño, unión fría y porosidad.

Como consecuencia de estos resultados, la producción por inyección convencional de aleaciones hipereutécticas queda limitada a cantidades de un $17-20 \% \mathrm{Si}$ en masa. El incremento del contenido de silicio origina otros problemas (además de las pésimas propiedades mecánicas derivadas de la microestructura) relacionados con las elevadas tem-
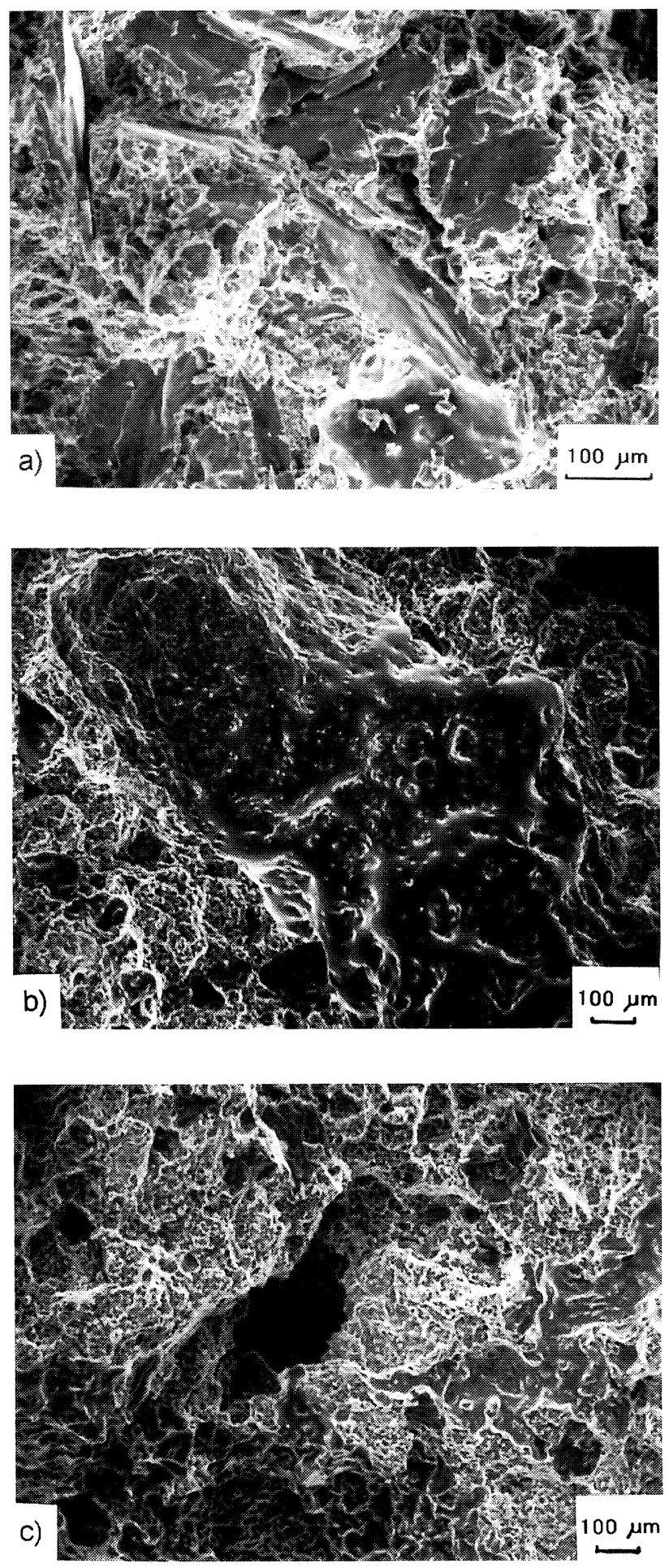

FIG. 1.- Superficies de fractura de las aleaciones convencionales. a) Partículas de gran tamaño. Al-36\%Si. b) Unión fría. c) Porosidad.

FIG. 1.- Fracture surface of conventional alloys. a) Big particles. Al-36\%Si. b) Cold shut. c) Porosity.

peraturas requeridas para su procesamiento: problemas con el desgaste del molde, pérdida de eficacia de los lubricantes y choque térmico del molde. 


\subsubsection{Nuevos composites de matriz metálica}

\subsubsection{Aleaciones atomizadas y extruidas}

Los resultados de los ensayos de tracción de las aleaciones $\mathrm{Al}-25 \% \mathrm{Si}-5 \% \mathrm{Cu}, \mathrm{Al}-30 \% \mathrm{Si}-5 \% \mathrm{Cu}$ y $\mathrm{Al}-25 \% \mathrm{Si}-5 \% \mathrm{Cu}-2 \% \mathrm{Mg}$ atomizadas y extruidas se muestran en la tabla II, incluyéndose en la misma las fracciones volumétricas de partículas de silicio primario (2). Se puede observar que, a pesar de la diferente fracción en volumen de las distintas aleaciones, el límite elástico es muy similar en todas ellas. Esto contradice los resultados obtenidos en diferentes composites, según los cuales fracciones en volumen de partículas superiores dan lugar a límites elásticos mayores (3).

La explicación para un límite elástico similar, para cantidades de silicio superiores, se puede atribuir a posibles fenómenos, más o menos extensos, de recristalización durante la extrusión de las aleaciones. El efecto de la solidificación rápida y de los diferentes coeficientes de expansión térmica entre el aluminio y el silicio da lugar a una elevada densidad de dislocaciones. La densidad de dislocaciones depende de la fracción en volumen de partículas según la expresión (9):

$$
\rho=\frac{B \cdot \varepsilon \cdot V_{\mathrm{f}}}{b \cdot d\left(1-V_{\mathrm{f}}\right)}
$$

donde $B$ es una constante, $V_{\mathrm{f}}$ es la fracción en volumen, $b$ es el vector de Burger, $d$ es el tamaño de partícula y $\epsilon$ es la diferencia en la deformación debido a los distintos coeficientes de expansión térmica. Según la expresión anterior, mayores fracciones en volumen de partículas dan lugar a una mayor

TABLA II.- Resultados de los ensayos de tracción de las aleaciones atomizadas y extruidas

TABLE II.- Tensile test results of as-sprayed and extruded alloys

\begin{tabular}{|l|c|c|c|c|}
\hline Aleación & $\begin{array}{c}\mathrm{Si}, \\
\% \text { vol. }\end{array}$ & $\begin{array}{c}\sigma_{\mathrm{Y}}, \\
\mathrm{MPa}\end{array}$ & $\begin{array}{c}\sigma_{\mathrm{UTS}}, \\
\mathrm{MPa}\end{array}$ & $\begin{array}{c}\epsilon_{\mathrm{p}} \\
\%\end{array}$ \\
\hline \multirow{3}{*}{$\mathrm{Al}-25 \% \mathrm{Si}-5 \% \mathrm{Cu}$} & 32 & 146 & 257,5 & 3,6 \\
& & 162 & 265 & 2,8 \\
& & 147 & 255 & 2,38 \\
\hline & \multirow{3}{*}{40} & 150 & 216,5 & 0,99 \\
& & 154 & 201,9 & 0,4 \\
& & 160 & 265 & 0,9 \\
\hline & 31,5 & 165 & 264 & 0,38 \\
& & 166 & 257 & 1,18 \\
\hline
\end{tabular}

densidad de dislocaciones. Esto, a su vez, origina una mayor fuerza impulsora para la recristalización. Por tanto, en la aleación Al-30\% $\mathrm{Si}-5 \% \mathrm{Cu}$, con respecto a la aleación $\mathrm{Al}-25 \% \mathrm{Si}-5 \% \mathrm{Cu}$, es de esperar que se produzca una mayor recristalización durante la extrusión. Este efecto contrarresta el aumento del límite elástico debido al incremento de la fracción en volumen de partículas.

El análisis fractográfico de las aleaciones atomizadas y extruidas indica que todas ellas presentan un aspecto muy similar. En la figura 2 se muestra una fractografía de la aleación $\mathrm{Al}-25 \% \mathrm{Si}-5 \% \mathrm{Cu}$, en las que se pueden ver partículas de silicio, en algunos casos, con grietas secundarias.

De la tabla II se puede observar, en las aleaciones $\mathrm{Al}-25 \% \mathrm{Si}-5 \% \mathrm{Cu}$ y $\mathrm{Al}-30 \% \mathrm{Si}-5 \% \mathrm{Cu}$, que un aumento de la cantidad de silicio da lugar a una disminución de la deformación plástica. Ello es debido a que el incremento de la fracción en volumen de partículas impide la deformación plástica de la matriz. No obstante, esto no va acompañado de un aumento de la resistencia a la tracción. Este resultado sugiere que a partir de cierta fracción en volumen de partículas se produce una incompatibilidad geométrica, por la cual las tensiones que se transmiten de la matriz a las partículas son lo suficientemente elevadas para que se produzca su fractura y ésta se transmita de una partícula a otra fácilmente debido a que se encuentran en contacto.

Por otra parte, la aleación $\mathrm{Al}-25 \% \mathrm{Si}-5 \% \mathrm{Cu}-$ $2 \% \mathrm{Mg}$ presenta, con relación a la misma aleación sin magnesio, un ligero aumento del límite elástico y una importante reducción en la deformación plástica. Este comportamiento debe ser atribuido al posible efecto endurecedor de la matriz de aluminio originado por la adición de magnesio, ya que tanto la fracción volumétrica como la distribución de

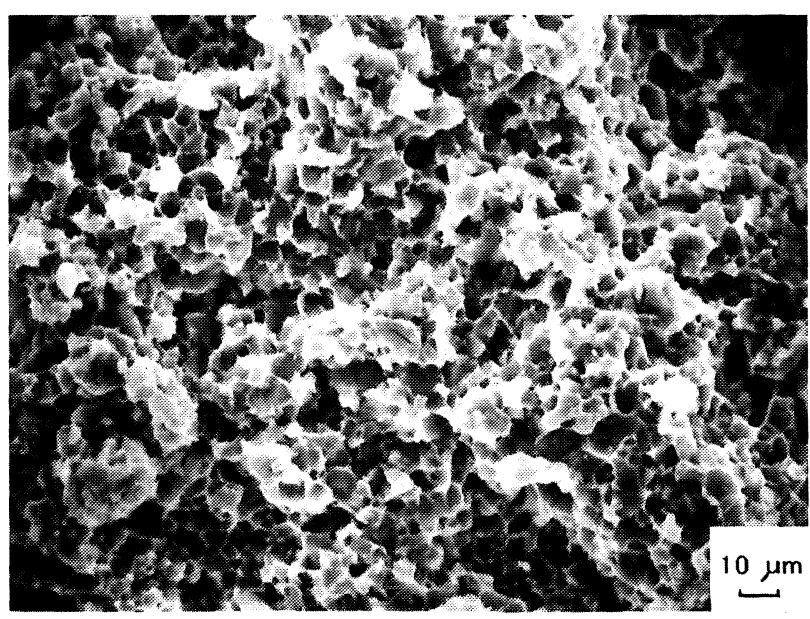

FIG. 2.- Superficie de fractura de la aleación Al$25 \% \mathrm{Si}-5 \% \mathrm{Cu}$. Atomizada y extruida.

FIG. 2.- Fracture surface Al-25\%Si-5\% Cu alloy as sprayed and extruded. 
tamaños de las partículas de silicio es prácticamente similar en ambos casos (2).

\subsubsection{Aleaciones conformadas en estado semisólido}

Los resultados de los ensayos de tracción de las aleaciones conformadas en estado semisólido presentan una cierta dispersión como consecuencia de los defectos (porosidad) o de las heterogeneidades microestructurales propias del proceso. Puesto que las propiedades de los composites dependen fuertemente de los parámetros microestructurales, la obtención de unas propiedades aceptables requiere un control estricto del proceso. En la tabla III se muestran los resultados de los ensayos de tracción de las aleaciones ensayadas, en las que se incluyen las fracciones volumétricas de partículas de silicio (2). En el caso de la aleación $\mathrm{Al}-25 \% \mathrm{Si}-5 \% \mathrm{Cu}-$ $2 \% \mathrm{Mg}$ se obtiene una elevada dispersión de los resultados debido a que presenta una mayor cantidad de porosidad.

Como en el caso de las aleaciones atomizadas y extruidas, se produce una disminución de la deformación plástica con el incremento de la cantidad de silicio, para las aleaciones $\mathrm{Al}-25 \% \mathrm{Si}-5 \% \mathrm{Cu}$ y $\mathrm{Al}-$ $30 \% \mathrm{Si}-5 \% \mathrm{Cu}$. En comparación con las aleaciones atomizadas y extruidas, se produce una caída de la deformación plástica en todas las aleaciones y un aumento de la resistencia a la tracción. En esta última, el efecto de la elevada fracción en volumen de partículas, junto a la influencia de los defectos existentes, produce una fractura prematura del material en el tramo ascendente de la curva tensión-deformación, por lo que no se alcanza el límite elástico.

TABLA III.- Resultados de los ensayos de tracción de las aleaciones conformadas en estado semisólido

TABLE III.- Tensile test results of semi-solid forming alloys

\begin{tabular}{|l|c|c|c|c|}
\hline \multicolumn{1}{|c|}{ Aleación } & $\begin{array}{c}\% \\
\text { vol. }\end{array}$ & $\begin{array}{c}\sigma_{\mathrm{Y}}, \\
\mathrm{MPa}\end{array}$ & $\begin{array}{c}\sigma_{\mathrm{UTS}} \\
\mathrm{MPa}\end{array}$ & $\begin{array}{c}\epsilon_{\mathrm{p}}, \\
\%\end{array}$ \\
\hline & & 274 & 314 & 0,53 \\
$\mathrm{Al}-25 \% \mathrm{Si}-5 \% \mathrm{Cu}$ & 28,5 & - & 255 & 0,16 \\
& & 262 & 295 & 0,4 \\
\hline & & - & 227,5 & 0,13 \\
& & - & 226,5 & 0,09 \\
$\mathrm{Al}-30 \% \mathrm{Si}-5 \% \mathrm{Cu}$ & 32,8 & - & 210,5 & - \\
\hline & & - & 331 & - \\
$\mathrm{Al}-25 \% \mathrm{Si}-5 \% \mathrm{Cu}-2 \% \mathrm{Mg}$ & 25 & - & 261 & - \\
& & - & 213 & - \\
\hline
\end{tabular}

La aleación $\mathrm{Al}-25 \% \mathrm{Si}-5 \% \mathrm{Cu}-2 \% \mathrm{Mg}$ es la que mayor resistencia presenta, aunque tampoco se ha alcanzado el límite elástico. Este resultado es atribuible al menor tamaño de partículas con respecto a las demás aleaciones conformadas en semisólido: para fracciones de partículas similares se obtienen resistencias superiores en composites con tamaños de partículas menores (3). Este resultado es válido si se comparan composites con la misma historia termomecánica: la comparación con las aleaciones atomizadas y extruidas no es válida puesto que su procesamiento es diferente. La dispersión en los valores obtenidos en esta aleación se debe a la presencia de una mayor cantidad de porosidad.

El estudio fractográfico de las aleaciones conformadas en semisólido muestra pequeñas diferencias entre las aleaciones. Las aleaciones $\mathrm{Al}-25 \% \mathrm{Si}$ $5 \% \mathrm{Cu}$ y $\mathrm{Al}-30 \% \mathrm{Si}-5 \% \mathrm{Cu}$ presentan gran cantidad de partículas de silicio en la superficie de fractura, con grietas secundarias en muchos casos, tal y como queda reflejado en la figura 3.
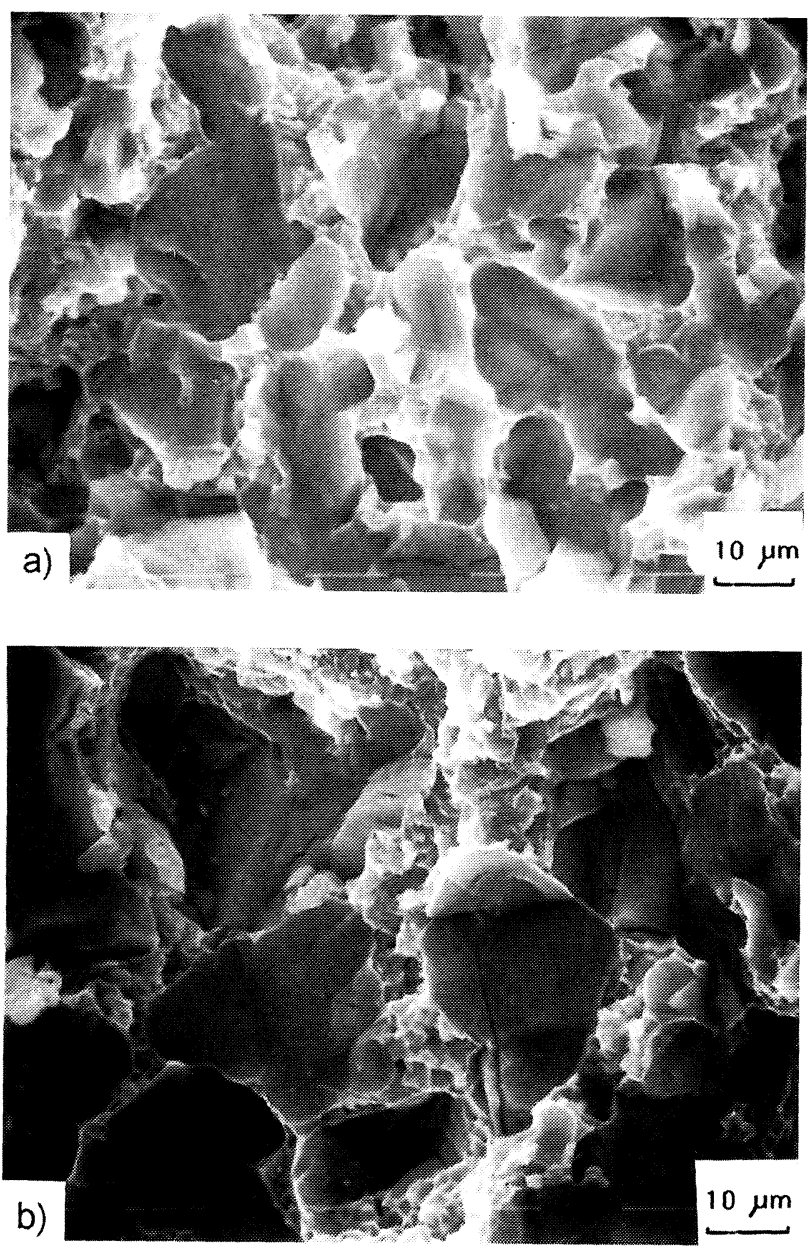

FIG. 3.- Superficie de fractura de las aleaciones conformadas en estado semisólido. a) $\mathrm{Al}-25 \% \mathrm{Si}$ $5 \% \mathrm{Cu}$. b) $\mathrm{Al}-30 \% \mathrm{Si}-5 \% \mathrm{Cu}$.

FIG. 3.- Fracture surface of a) $\mathrm{Al}-25 \% \mathrm{Si}-5 \% \mathrm{Cu}$ thixoformed alloy. b) Al-30\%Si-5\% Cu. 
Como se ha dicho con anterioridad, la presencia de porosidad o heterogeneidades en la microestructura origina cierta dispersión en los resultados. La aleación $\mathrm{Al}-25 \% \mathrm{Si}-5 \% \mathrm{Cu}$, que es la que mayor deformación presenta, muestra pequeñas diferencias en su superficie de fractura, dependiendo de su deformación plástica: en las probetas que han dado mayor deformación macroscópica se observan las partículas de silicio rodeadas por hilos o pequeñas cavidades dúctiles; en cambio, cuando la deformación macroscópica ha sido menor aparecen agrupamientos de partículas con pocos indicios de microplasticidad, como se puede ver en la figura 4.

La superficie de fractura de la aleación $\mathrm{Al}-25 \% \mathrm{Si}$ $5 \% \mathrm{Cu}-2 \% \mathrm{Mg}$ es ligeramente diferente a las anteriormente comentadas. Como se puede apreciar en la figura 5, esta aleación presenta muy poca microplasti-

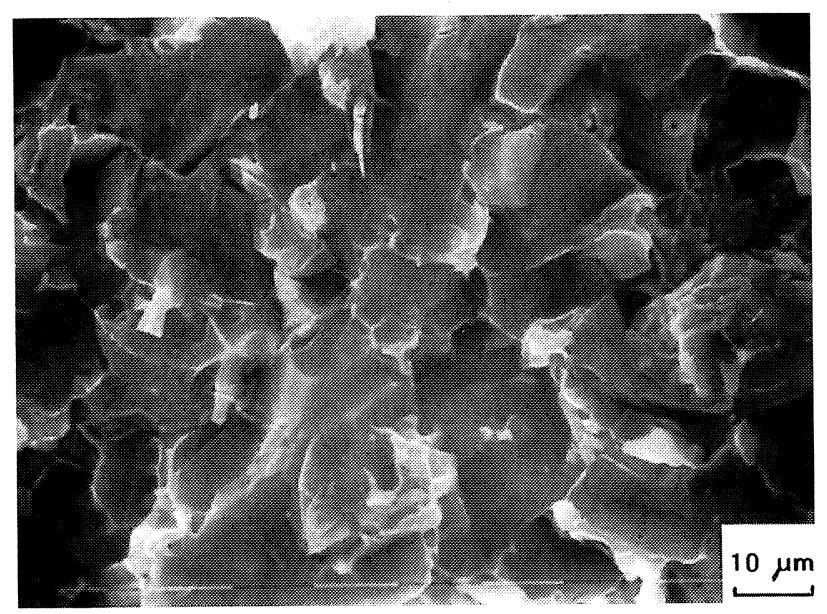

FIG. 4.- Superficie de fractura de la aleación Al$25 \% \mathrm{Si}-5 \% \mathrm{Cu}$ conformada en estado semisólido. Aglomeración de particulas de silicio.

FIG. 4.- Fracture surface of Al-25\%Si-5\% Cu thixoformed. Cluster of silicon particles.

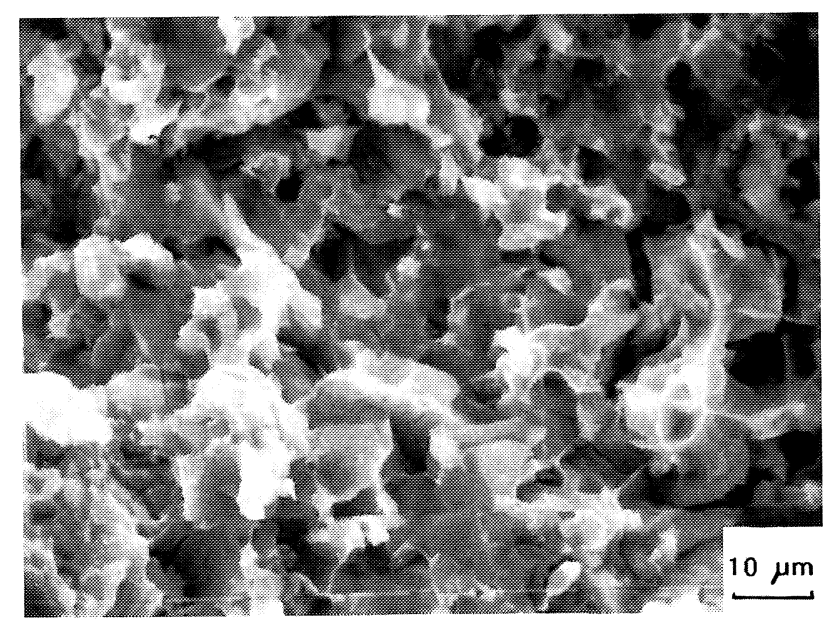

FIG. 5.- Superficie de fractura de la aleación Al$25 \% \mathrm{Si}-5 \% \mathrm{Cu}-2 \% \mathrm{Mg}$ conformada en estado semisólido.

\section{FIG. 5.- Fracture surface of Al-25\%Si-5\% Cu-} $2 \% \mathrm{Mg}$ thixoformed alloy.

cidad, de acuerdo a su menor deformación macroscópica. También presenta una superficie de fractura, macroscópicamente, bastante menos rugosa que en el caso de la aleación $\mathrm{Al}-25 \% \mathrm{Si}-5 \% \mathrm{Cu}$.

Los mecanismos de fractura son los mismos en todas las aleaciones: la fractura se inicia en las partículas de silicio, se transmite hacia las zonas intercelulares y, finalmente, se produce la fractura dúctil a través del aluminio. En ningún caso se ha observado fractura a través de la intercara entre las partículas y la matriz, lo que significa que su resistencia es mayor que la resistencia de las partículas de silicio.

El hecho de que el proceso de fractura venga dominado por la fractura de las partículas de silicio, está apoyado por el estudio del perfil de las superficies de fractura. En la figura 6 se muestra un perfil

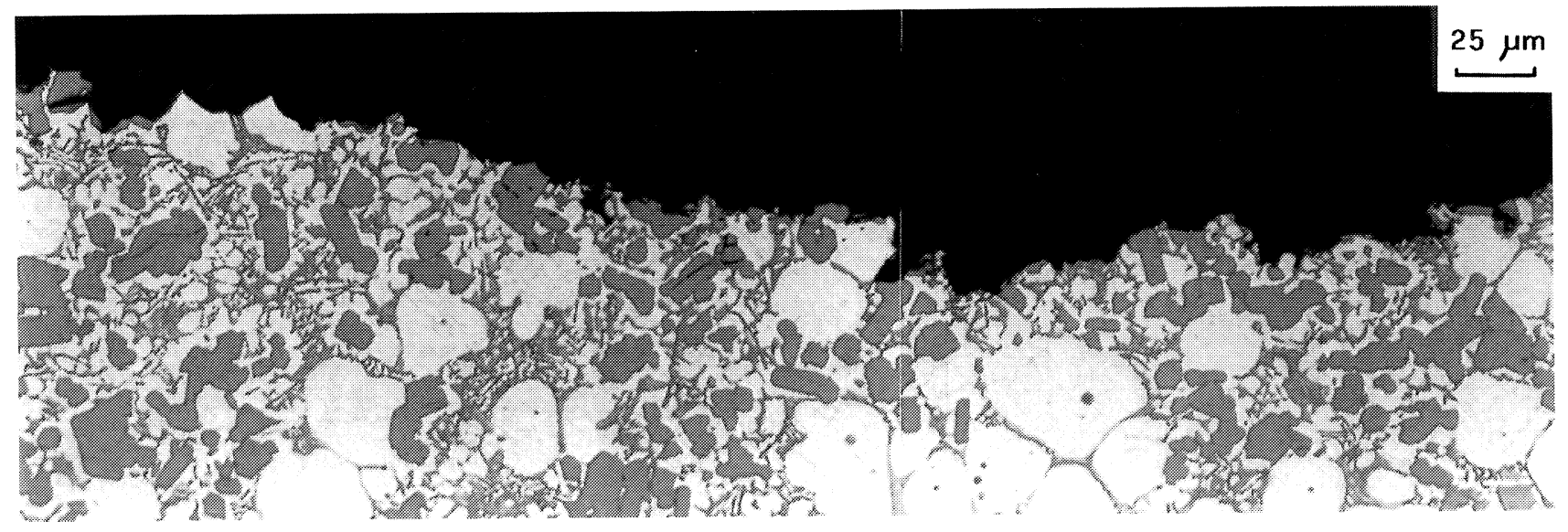

FIG. 6.- Perfil de la superficie de fractura en la aleación Al-25\%Si-5\% Cu conformada en estado semisólido. La grieta se propaga preferentemente a través de las partículas de silicio y del intermetálico $\mathrm{CuAl}_{2}$.

FIG. 6.- Crack profile of Al-25\%Si-5\% Cu thixoformed alloy: crack propagates across silicon particles and $\mathrm{CuAl}_{2}$ intermetallic. 
de fractura de la aleación $\mathrm{Al}-25 \% \mathrm{Si}-5 \% \mathrm{Cu}$ conformada en estado semisólido, en la que se puede observar gran cantidad de partículas de silicio, zonas intercelulares y algunos glóbulos de aluminio muy deformados. El daño se concentra en la superficie de fractura, no extendiéndose por debajo de ella.

La medida de la cantidad de silicio es, aproximadamente, dos veces superior a la cantidad existente en la zona adyacente al perfil de fractura (1). Esto confirma que la fractura se produce principalmente a través de las partículas de silicio.

3.1.2.3. Comportamiento de la aleación $\mathrm{Al}-25 \% \mathrm{Si}$ $5 \% \mathrm{Cu}$ antes y después del conformado en estado semisólido

En la figura 7 se representan las curvas de tensión real frente a deformación real de la aleación $\mathrm{Al}-25 \% \mathrm{Si}-5 \% \mathrm{Cu}$ antes y después del conformado en estado semisólido. Se observa una clara diferencia entre el comportamiento de la misma aleación en ambos estados, tal y como se ha podido observar comparando los datos de las tablas II y III.

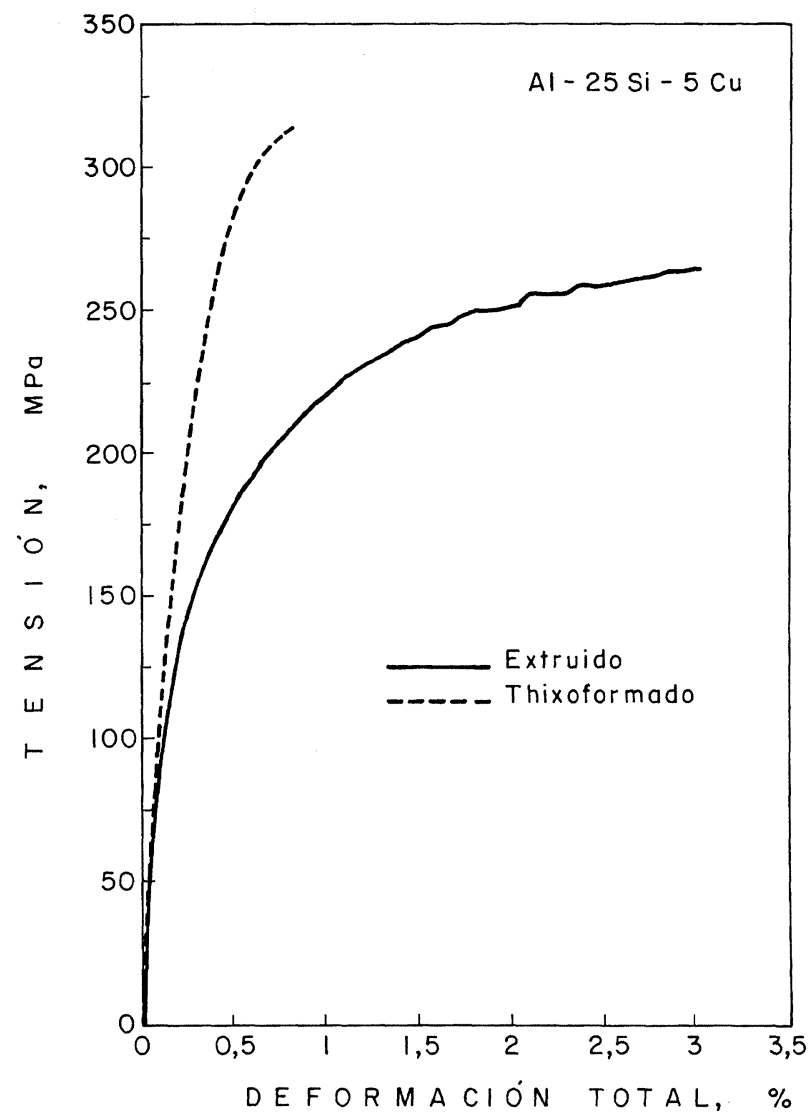

FIG. 7.- Diferencia en el comportamiento de la aleación $\mathrm{Al}-25 \% \mathrm{Si}-5 \% \mathrm{Cu}$ antes y después del conformado en estado semisólido.

FIG. 7.-Different behaviour of Al-25\%Si-5\% Cu before and after thixoforming.
La diferencia en el comportamiento se atribuye a la distinta microestructura en ambas condiciones, de las que se muestran micrografías en la figura 8 (2). Mientras en el material atomizado y extruido, las partículas de silicio se encuentran homogéneamente distribuidas en la matriz, en la aleación conformada en semisólido se tiene una microestructura celular (2) a la que se considera responsable del notablemente superior límite elástico.

Esta peculiar distribución de las fases sugiere que se puede producir un endurecimiento semejante al endurecimiento por juntas de grano. Según este tipo de endurecimiento, el movimiento de las dislocaciones durante la deformación plástica queda bloqueado por las juntas de grano. En las aleaciones presentes, la especial distribución de las partículas de silicio y de la fase intermetálica $\mathrm{CuAl}_{2}$, colaborando ambas en la formación de la estructura celular, actúa de la misma manera, tal y como se representa esquemáticamente en la figura 9. El número de dislocaciones que pueden soportarse depende del
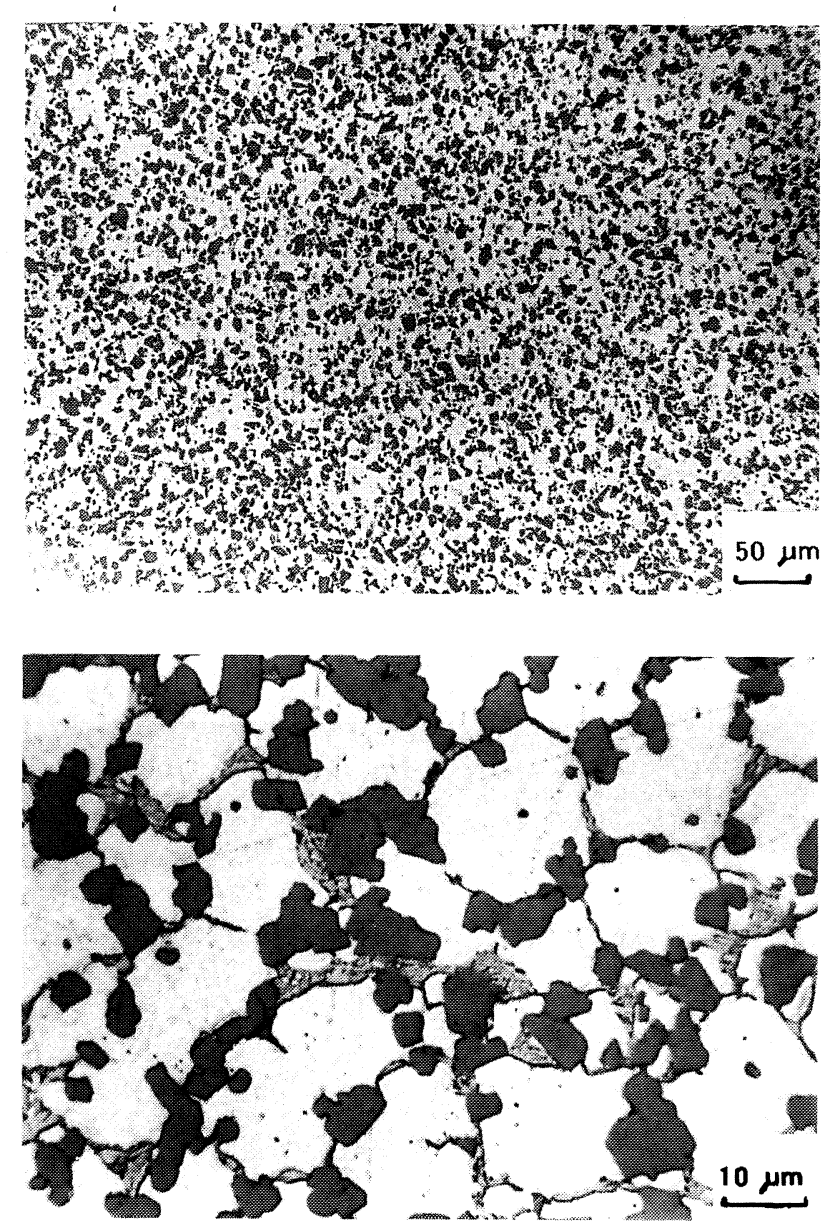

FIG. 8.- Microestructura de la aleación Al-25\%Si$5 \% \mathrm{Cu}$ antes y después del conformado en estado semisólido. Estructura celular.

FIG. 8.- Microstructure of Al-25\%Si-5\% Cu alloy before and after thixoforming. Cellular structure. 


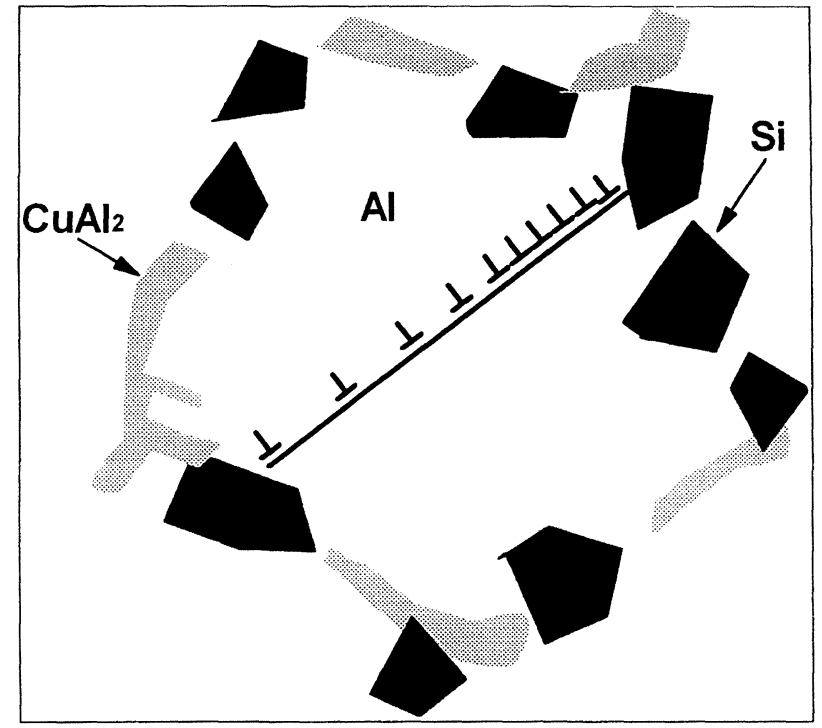

FIG. 9.- Esquema del mecanismo de endurecimiento operante en estos composites, similar al mecanismo de endurecimiento de límite de grano.

FIG. 9.- Schematic figure showing the strengthening operating on this composites, similar to the conventional grain boundary strengthening.

tipo de barrera, de la orientación entre el plano de deslizamiento y las características microestructurales que actúan como barrera, del material y de la temperatura (10).

Debido a los parámetros de procesamiento, la microestructura se presenta más o menos celular. Las probetas que tienen más carácter celular han dado lugar a un superior límite elástico y resistencia a la tracción, lo que confirma el tipo de endurecimiento propuesto.

El hecho de que el material atomizado y extruido no presente estructura celular da lugar a un límite elástico menor en todas las aleaciones, debido a la mayor facilidad para acomodar la deformación plástica en la matriz.

\subsubsection{Comparación con composites de aleaciones de aluminio con partículas de $\mathrm{SiC}$}

$\mathrm{Al}$ intentar comparar las propiedades obtenidas mediante los ensayos de tracción con las reportadas por investigadores que han trabajado con composites similares, surgen varias dificultades. En primer lugar, es necesario tener en cuenta el tipo de aleaciones de matriz utilizadas por estos investigadores: normalmente se eligen aleaciones de aluminio de alta resistencia o susceptibles de ser tratadas térmicamente para modificar su resistencia. Otro aspecto a tener en cuenta es el tipo de partículas utilizadas, generalmente $\mathrm{SiC}$, que tiene una resistencia mayor que el silicio y se introduce de tamaño y forma determinados. Adicionalmente, es posible controlar la cantidad de defectos (y, por tanto, la resistencia) que presentan las partículas, según el tipo de tecnología utilizada para su fabricación. Finalmente, y quizás como punto más importante, es obligado tener en cuenta el tipo de procesamiento utilizado para la fabricación del composite (metalurgia de polvos, distintos tipos de inyección, forja líquida, algún proceso de solidificación rápida, etc.). Tanto el proceso como las variables del proceso influyen enormemente en la calidad y propiedades mecánicas finales del material, como se ha podido comprobar durante el procesamiento de las presentes aleaciones hipereutécticas: pequeños cambios en la temperatura dan lugar a fuertes cambios en el aspecto de la matriz; la presencia de defectos (poros, unión fría) depende también de las variables elegidas.

Por otro lado y dentro del tipo de procesamiento, se ha querido comparar con composites producidos mediante tecnologías semejantes, que den lugar a piezas semiacabadas y no se requiera un costoso mecanizado posterior. Este aspecto es muy importante a la hora de la utilización de estos materiales a nivel industrial, puesto que el mecanizado de los composites es dificultoso debido a la presencia de partículas muy abrasivas y en cantidades elevadas.

Según este planteamiento, se han buscado en la bibliografía composites producidos mediante técnicas similares, como la metalurgia líquida, que dan lugar a defectos parecidos. Los valores encontrados son similares a los obtenidos en los nuevos composites basados en aleaciones hipereutécticas de Al$\mathrm{Si}$, aunque hay bastante dispersión en los resultados, a pesar de tratarse de sistemas muy parecidos (11-16). Obviamente, la comparación entre los composites estudiados en este trabajo y los basados en aleaciones de aluminio reforzadas con partículas de $\mathrm{SiC}$ no es totalmente válida, puesto que parámetros tales como fracción en volumen, tamaño y estado superficial de las partículas, tratamiento térmico de los composites obtenidos o distribución de las partículas en la matriz, pueden dar lugar a propiedades mecánicas muy diferentes.

\subsection{Ensayos de tracción a elevada temperatura}

\subsubsection{Aleaciones conformadas en semi-sólido}

En la figura 10 se representa la variación de la resistencia a la tracción y de la ductilidad con la temperatura de las aleaciones $\mathrm{Al}-25 \% \mathrm{Si}-5 \% \mathrm{Cu}, \mathrm{Al}-$ $30 \% \mathrm{Si}-5 \% \mathrm{Cu}$ y $\mathrm{Al}-25 \% \mathrm{Si}-5 \% \mathrm{Cu}-2 \% \mathrm{Mg}$.

Como puede observarse, la aleación que mejores propiedades ofrece a elevada temperatura es la $\mathrm{Al}-$ $25 \% \mathrm{Si}-5 \% \mathrm{Cu}-2 \% \mathrm{Mg}$, manteniendo una resistencia a la tracción, a $300{ }^{\circ} \mathrm{C}$, de $265 \mathrm{MPa}$. 

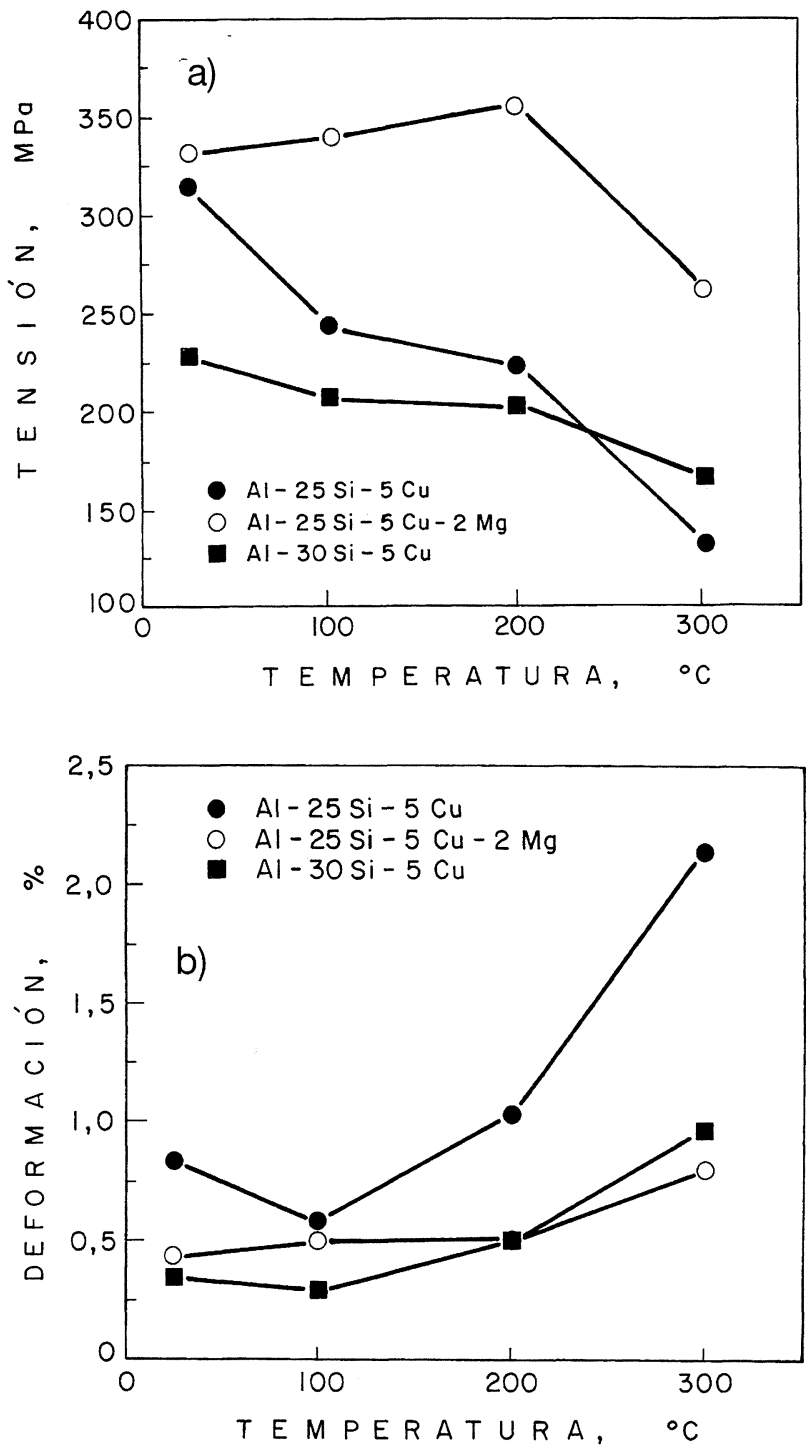

Fig. 10.- Variación con la temperatura para las tres aleaciones estudiadas conformadas en estado semisólido. a) Resistencia mecánica. b) Ductilidad.

FIG. 10.-Variation with temperature of three thixoformed alloys. a) Tensile strength.

\section{b) Deformation.}

La aleación Al-30\%Si-5\% Cu se comporta a 300 ${ }^{\circ} \mathrm{C}$ ligeramente mejor que la aleación $\mathrm{Al}-25 \% \mathrm{Si}$ $5 \% \mathrm{Cu}$, lo que parece indicar que a altas temperaturas el efecto de la fracción en volumen de partículas sí tiene el efecto esperado, impidiendo la deformación plástica de la matriz.

En todas las aleaciones el modo dominante de fractura sigue siendo la rotura de las partículas, aunque la matriz juega un papel más importante que se traduce en un aumento de la deformación macroscópica. La cantidad de partículas en las superficies de fractura sigue siendo muy elevada, tal y como queda reflejado en la figura 11 en la que se muestra la aleación $\mathrm{Al}-25 \% \mathrm{Si}-5 \% \mathrm{Cu}$ ensayada a
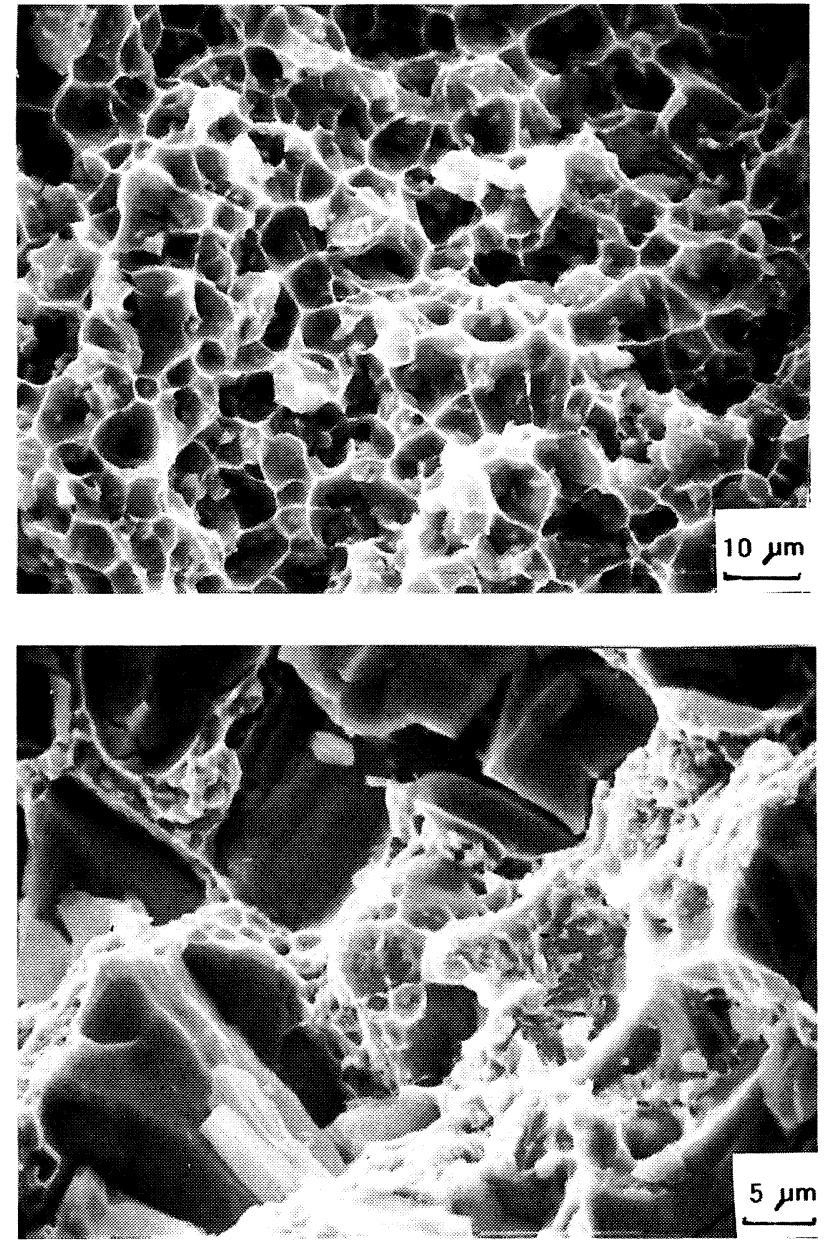

FIG. 11.- Superficie de fractura de la aleación Al$25 \% \mathrm{Si}-5 \% \mathrm{Cu}$ ensayada a $300{ }^{\circ} \mathrm{C}$ antes y despues del conformado en estado semisólido.

FIG. 11.-Surface fracture of Al-25\%Si-5\% Cu alloy tested at $300^{\circ} \mathrm{C}$, before and after thixoforming.

$300{ }^{\circ} \mathrm{C}$. La fractografía refleja el aumento de la deformación macroscópica que experimenta el material con la temperatura: existe una gran cantidad de cavidades dúctiles que rodean las partículas de silicio.

La observación del perfil de fractura de la aleación muestra una gran cantidad de daño debajo del perfil de fractura, contrariamente a lo observado a temperatura ambiente. En la figura 12 se muestra la sección longitudinal de esta aleación en una probeta ensayada a $300^{\circ} \mathrm{C}$; aparece una elevada cantidad de partículas fracturadas, alejadas de la superficie de fractura. Este resultado se debe a que con la temperatura se relajan las tensiones en la matriz, la carga que se transmite de la matriz a las partículas es menor y se obtiene una deformación macroscópica mayor, hasta que, al aumentar la deformación plástica se alcanza la carga que produce la fractura de las partículas. Esta carga depende del tamaño, la forma y la orientación de las partículas. 


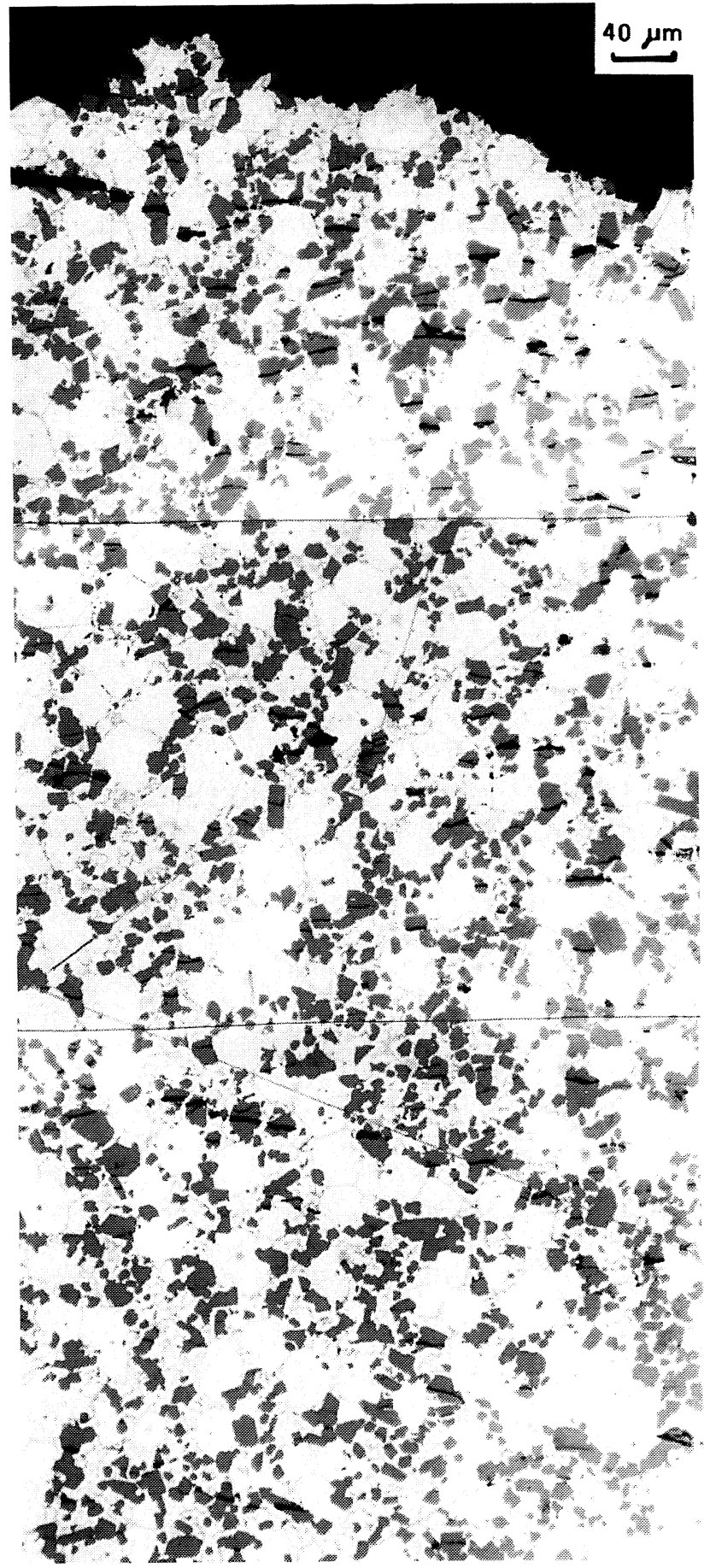

FIG. 12.- Perfil de fractura de la aleación Al$25 \% \mathrm{Si}-5 \% \mathrm{Cu}$ conformada en estado semisólido y ensayada a $300{ }^{\circ} \mathrm{C}$. Particulas agrietadas lejos de la superficie de fractura.

FIG. 12.- Crack profile of $\mathrm{Al}-25 \% \mathrm{Si}-5 \% \mathrm{Cu}$ thixoformed alloy tested at $300{ }^{\circ} \mathrm{C}$. Cracked particles far from the fracture surface.

\subsubsection{Comportamiento de la aleación Al-25\%Si- $5 \% \mathrm{Cu}$ antes y después del conformado en estado semisólido}

Del mismo modo que a temperatura ambiente, la aleación conformada en semisólido presenta una resistencia a la tracción y un límite elástico notablemente superiores a la aleación atomizada y extruida. En la figura 13 se muestra la variación de la resistencia, del límite elástico y de la deformación con la temperatura, para la aleación Al-25\%Si$5 \% \mathrm{Cu}$ antes y después del conformado en semisólido. Lo más llamativo es el importante aumento de la deformación macroscópica que experimenta la aleación en el estado atomizado y extruido.

Una vez más, la explicación a estos resultados se encuentra en el efecto de la estructura celular de la aleación conformada en semisólido que tiene el mismo efecto que a temperatura ambiente. En la aleación atomizada y extruida el proceso de fractura viene dominado por la deformación de la matriz y
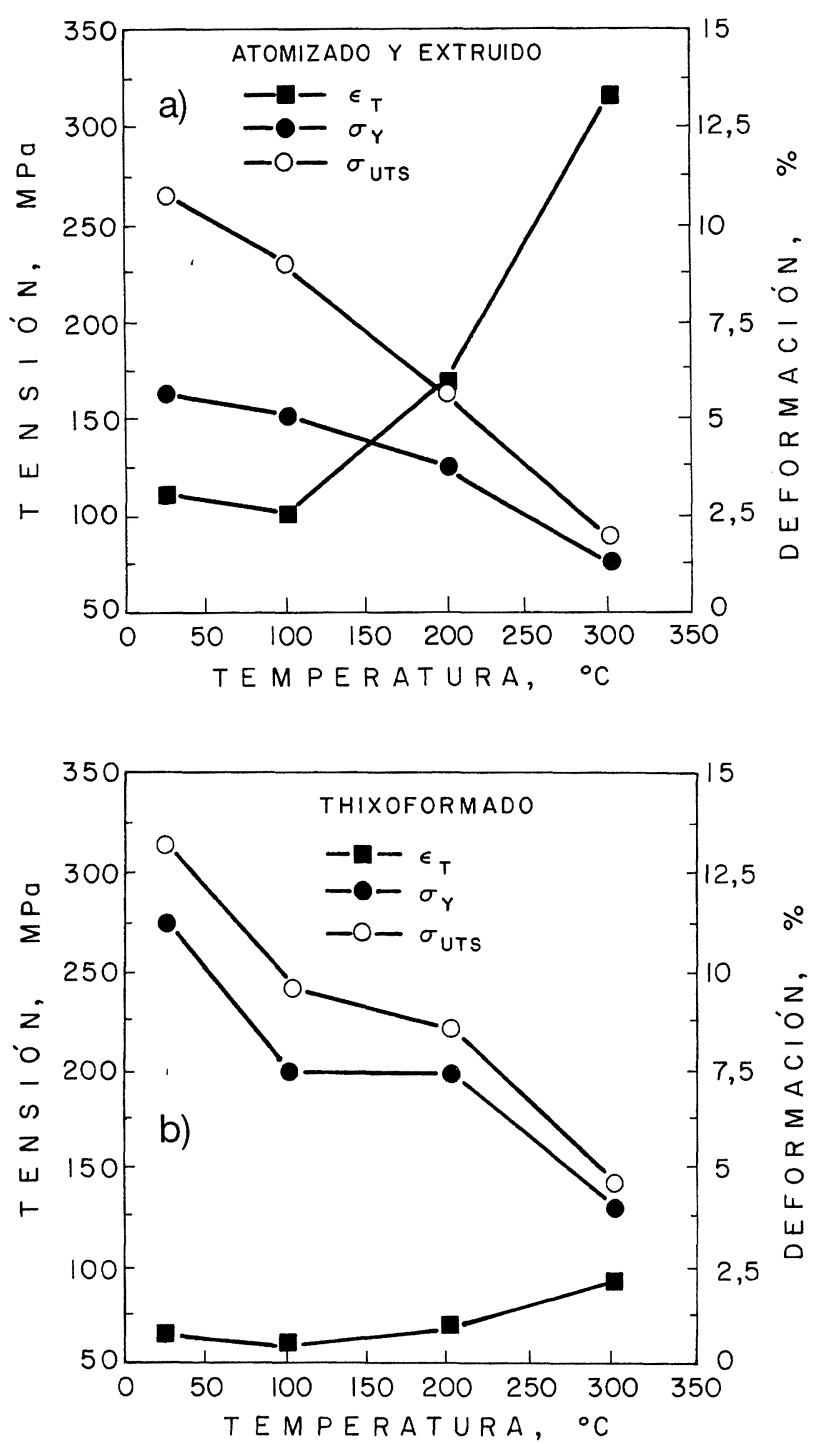

FIG. 13.- Aleación Al-25\%Si-5\%Cu. Variación de las propiedades con la temperatura. a) Atomizada y extruida. b) Conformada en estado semisólido.

FIG. 13.- Al-25\%Si-5\% Cu alloy. Variation of the properties with the temperature. a) As-sprayed and extruded. b) As-thixoformed. 
esto explica la elevada deformación plástica que experimenta el material.

\subsubsection{Comparación con composites de aleaciones de aluminio con partículas de $\mathrm{SiC}$}

Cuando se comparan las propiedades de los composites basados en aleaciones hipereutécticas de Al-Si con composites de aleaciones de aluminio reforzados con partículas cerámicas, una clara observación es que los composites de Al-Si mantienen mejor las propiedades al aumentar la temperatura. En los composites reforzados con partículas cerámicas se ha encontrado experimentalmente que existe una temperatura de transición a partir de la cual cambia el modo de fractura del material de fractura a través de las partículas a fractura en la matriz, por debilidad de la intercara (17 y 18). Luster et al. (19), observan que el composite $6061+20 \% \mathrm{Al}_{2} \mathrm{O}_{3}$ no presenta mejores propiedades que la aleación de la matriz a elevadas temperaturas.

Como ejemplo de la fuerte caída de la resistencia a la tracción con la temperatura, se encuentra un composite de $6090+25 \% \mathrm{SiC}$ (producido por metalurgia de polvos) reportado por Nieh et al. (20). Este material tiene un valor de $\sigma_{\text {UTS }}$ a temperatura ambiente de $541 \mathrm{MPa}$, y a $300{ }^{\circ} \mathrm{C}$, la resistencia baja hasta $126 \mathrm{MPa}$ (que es el $23 \%$ de la resistencia a temperatura ambiente). En el caso de la aleación $\mathrm{Al}-25 \% \mathrm{Si}-5 \% \mathrm{Cu}$ conformada en estado semisólido, la resistencia a la tracción a temperatura ambiente es de $314 \mathrm{MPa}$, y a $300{ }^{\circ} \mathrm{C}$ es de 143,5 $\mathrm{MPa}$ (el $46 \%$ de la resistencia a temperatura ambiente).

\section{CONCLUSIONES}

- Mediante una combinación de un proceso de solidificación rápida y del conformado en estado semisólido, es posible obtener aleaciones hipereutécticas de Al-Si con un comportamiento a tracción superior a las aleaciones producidas convencionalmente.

- La aleación Al-25\%Si-5\% Cu, conformada en estado semisólido, es la que presenta una mayor deformación a la fractura. La aleación Al$25 \% \mathrm{Si}-5 \% \mathrm{Cu}-2 \% \mathrm{Mg}$, con partículas de menor tamaño, es la que mayor resistencia a la tracción presenta.

- Todas las aleaciones presentan una menor deformación a la fractura y una resistencia a la tracción mayor después del conformado en estado semisólido. Este resultado se atribuye a la estructura celular que presentan las aleaciones en esta condición.

- La aleación $\mathrm{Al}-25 \% \mathrm{Si}-5 \% \mathrm{Cu}-2 \% \mathrm{Mg}$ mantiene muy buenas propiedades hasta la temperatura de $300^{\circ} \mathrm{C}$.

- El modo de fractura es el mismo a temperatura ambiente y a elevadas temperaturas: se fracturan en primer lugar las partículas de silicio. No se produce en ningún caso fractura a través de la intercara entre las partículas y la matriz. Este modo de fractura se presenta frecuentemente en los composites de aleaciones de aluminio reforzados con $\mathrm{SiC}$.

\section{REFERENCIAS}

(1) Valer Goñi, J., Rodriguez Ibabe, J.M. y Urcola J.J. Scr. Mater., 34 (3), 1996: 483-489.

(2) Valer, J., Rodriguez Ibabe, J.M., Urcola J.J. Rev. Metal., 32 (4), 1996: 231-247.

(3) Lloyd, D.J. Int. Mater. Rev., 39 (1), 1994: 1-23.

(4) Arsenault, R.J., Fishman, S. y Taya, M. Prog. Mater. Sci., 38, 1994: 1-157.

(5) You, C.P., Thompson, A.W. y Berstein, I.M. Scr. Metall., 21, 1987: 181-184.

(6) Lloyd, D.J., Lagacé, H., Mcleod, A. y Morris, P.L. Mater. Sci. Eng., A107, 1989: 73-80.

(7) Mondolfo, L.F. Aluminium alloys: structure and microstructure, Butterworth and Co., Londres, 1976.

(8) Ghomashchi, M.R. Scand. J. Met., 22, 1993: 61-67.

(9) Wu, Y.W., Cassada, W.A. y Lavernia, E.J., Metall. Trans., 26A, 1995: 1.235-1.247.

(10) Arsenault, R.J. y Shi, N. Mater. Sci. Eng., A81, 1986: 175-187.

(11) Wang, Z. y Zhang, R.J. Metall. Trans., 22A, 1991: 1.585-1.593.

(12) KIM, Y.-H, LeE, S., Kim, N.J. y CHo, K.-M. Scr. Metall. Mater., 31 (12), 1994: 1.629-1.634.

(13) Kamat, S.V., Hirth, J.P. y Mehrabian, R. Acta Metall. Mater., 37 (9), 1989: 2.395-2.402.

(14) Vedani, M., Piatti, G. y Di Gregorio, C. Mater. Sci. Technol., 10, 1994: 132-140.

(15) SAmuel, F.H. y Samuel, A.M. Metall. Trans., 25A, 1994: 2.247-2.263.

(16) Samuel, A.M. y Samuel, F.H. J. Mater. Sci., 30, 1995: 1.698-1.708.

(17) Syu, D.-G.C. y Ghosh, A.K. Mater. Sci. Eng., A184, 1994: 27-35.

(18) Zhao, D., Tuler, F.R. y Lloyd, D.J. Acta Metall. Mater., 42 (7), 1994: 2.525-2.533.

(19) Luster, J.W., Thurman, M. y Baumann, R. Mater. Sci. Technol., 9, 1993: 853-862.

(20) Nieh, T.G., Luster, D.R. y Syn, C.K. Scr. Metall. Mater., 32 (5), 1995: 707-712. 